\title{
DSMC simulations of Turing patterns in concentrated growing systems
}

Cite as: AIP Conference Proceedings 2132, 070008 (2019); https://doi.org/10.1063/1.5119562

Published Online: 05 August 2019

Gabriel Morgado, Bogdan Nowakowski, and Annie Lemarchand

ARTICLES YOU MAY BE INTERESTED IN

Validation of DSMC and NS computations for high-enthalpy non-equilibrium flows in ground and flight tests

AIP Conference Proceedings 2132, 070007 (2019); https://doi.org/10.1063/1.5119561

A microscopic study of one-dimensional $\mathrm{H}_{2}-\mathrm{O}_{2}$ detonation using DSMC method AIP Conference Proceedings 2132, 070013 (2019); https://doi.org/10.1063/1.5119567

Monte Carlo simulation of a gas transport system based on thermal induced flow AIP Conference Proceedings 2132, 070015 (2019); https://doi.org/10.1063/1.5119569

\section{Conference Proceedings}

Get $30 \%$ off all print proceedings!
Enter Promotion Code $P / D /=30$ at checkout

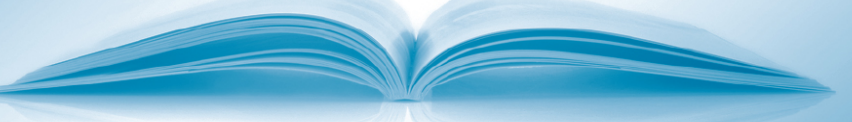




\title{
DSMC Simulations Of Turing Patterns In Concentrated Growing Systems
}

\author{
Gabriel Morgado ${ }^{1,2}$, Bogdan Nowakowski $^{1}$ and Annie Lemarchand ${ }^{2, a), b)}$ \\ ${ }^{1}$ Institute of Physical Chemistry, Polish Academy of Sciences, Kasprzaka 44/52, 01-224 Warsaw, Poland \\ ${ }^{2}$ Sorbonne Université, Centre National de la Recherche Scientifique CNRS, Laboratoire de Physique Théorique de la \\ Matière Condensée, LPTMC, 4 place Jussieu, case courrier 121, 75252 Paris Cedex 05, France \\ ${ }^{a)}$ Corresponding author: anle@lptmc.jussieu.fr \\ b)URL: https://www.lptmc.jussieu.fr/users/anle
}

\begin{abstract}
The control of self-organized structures such as Turing patterns is a challenge for chemical engineers. We propose a reaction-diffusion model that includes the reactive role of the solvent and admits Turing patterns of adaptable wavelength. Changing the concentration of the solute is sufficient to tune the wavelength. Particles dynamics simulations are successfully performed, illustrating wavelength control down to the submicrometric scale.
\end{abstract}

\section{INTRODUCTION}

The formation of a periodic spatial structure along an axis is a typical example of self-organisation encountered in biology in the plant kingdom as in the animal kingdom. Palm leaves, the segments of many insects or crustaceans, and the spine of vertebrates give some examples of structures resulting from axial segmentation. It is tempting to use models from biology in the context of chemical engineering, with the goal of designing periodic spatial structures by biomimetism. In particular, the model of morphogenesis introduced by Turing has the advantage of being compatible with a microscopic approach [1]. A model with a small number of elementary processes of reaction and diffusion type can be used to simulate submicrometric Turing patterns [2]. However, the wavelength of the structure is imposed by the dynamical parameters whereas an application to chemical engineering would require a flexible pattern with a tunable wavelength. We propose to control the value of the wavelength, simply by playing on the dilution of the system. Indeed, sufficiently far from the high dilution limit, the solvent can be explicitly involved in the reaction scheme, in such a way that varying the total concentration could be sufficient to tune the wavelength of Turing pattern [3]. Our goal is to show the possibility of controlling the wavelength of submicrometric Turing patterns by adapting the Direct Simulation Monte Carlo (DSMC) method to the simulation of concentrated solutions.

DSMC has been introduced as a direct simulation of the Boltzmann equation in order to efficiently simulate the dynamics of rarefied gases with large Knudsen numbers for which the continuous description is not valid. The principles of DSMC extend over the entire range of validity of the Boltzmann equation. We apply the Bird simulation method to a reactive system said to be concentrated because of the relatively high proportion of the solute with respect to the solvent. Hence, the departure from dilute conditions is characterized by the ratio of the solute density to the total density. However, the total density is supposed to remain sufficiently low to ensure that dynamics is correctly described in the framework of the Boltzmann equation. When binary reactive collisions are involved, the kinetic Monte Carlo simulation method is straightforwardly extended to reactive systems, the transition rates being deduced from the reactive collision integrals of the corresponding Boltzmann equation. We use a trick to simulate ternary reactions that already proved to be satisfactory $[4,5]$ : The trimolecular interaction is decomposed into a standard binary collision and reaction is accepted depending on the presence of the third reactive species in the neighborhood of the colliding pair. We refer to reference [6] for a detailed presentation of the adapted DSMC algorithm. As a result, the diffusion coefficients of the components and the rate constant of the ternary reaction depend on the total density and consequently the departure from dilute conditions, implying a scaling of the simulation results before the

31st International Symposium on Rarefied Gas Dynamics

AIP Conf. Proc. 2132, 070008-1-070008-6; https://doi.org/10.1063/1.5119562

Published by AIP Publishing. 978-0-7354-1874-5/\$30.00 
comparison with those of the macroscopic description.

In addition to simulating the Boltzmann equation, DSMC includes the description of the internal fluctuations. Using DSMC to simulate Turing patterns allows us to check the robustness of the spatial structure to fluctuations in small systems down to the nanoscale.

The paper is organized as follows. Section 2 is devoted to the presentation of the reaction-diffusion model including an explicit role for the solvent. Section 3 gives the specificities of DSMC algorithm used to reproduce a Turing pattern in a concentrated system. We give the results in section 4 . Section 5 contains conclusion.

\section{A MICROSCOPIC MODEL OF TURING PATTERNS WITH POSSIBLE SOLVENT ROLE}

We consider the following reaction scheme involving an activator A with a diffusion coefficient $D_{A}$, an inhibitor B with a larger diffusion coefficient $D_{B}>D_{A}$, and interaction with two reservoirs of chemical species or chemostats $\mathrm{R}_{1}$ and $\mathrm{R}_{2}$ which maintain the system far from equilibrium $[7,8,9]$ :

$$
\begin{aligned}
& \text { A } \stackrel{k_{1}}{\longrightarrow} \mathrm{R}_{1} \\
& 2 \mathrm{~A}+\mathrm{B} \stackrel{k_{2}}{\longrightarrow} 3 \mathrm{~A} \\
& \mathrm{~B} \underset{k_{-3}^{\prime}}{\stackrel{k_{3}}{\rightleftharpoons}} \mathrm{R}_{2}
\end{aligned}
$$

This system is known to be associated with reaction-diffusion equations admitting spatial structures of Turing type. As explained by Gierer and Meinhardt [10], stabilization of a Turing pattern requires local activation through the autocatalytic formation of the activator A and long-range inhibition through the fast-diffusive inhibitor $\mathrm{B}$. We already proved at the macroscopic scale that considering the solvent $\mathrm{S}$ as an actual reactant in a concentrated system according to

$$
\begin{aligned}
\mathrm{A}+\mathrm{S} & \longrightarrow \mathrm{R}_{1} \\
2 \mathrm{~A}+\mathrm{B} & \longrightarrow 3 \mathrm{~A} \\
\mathrm{~B}+\mathrm{S} & \rightleftharpoons \mathrm{R}_{2}
\end{aligned}
$$

leads to a Turing pattern whose wavelength can be tuned simply by varying the total concentration $C=A+B+S$, e.g. the quantity of solvent $\mathrm{S}$ at constant $A+B$ value [3]. In view of DSMC simulations, it is tempting to assign the role of reservoir particles to solvent particles. In particular, the first step given in Eq. (4) is replaced by $A+S \longrightarrow 2 S$, which interestingly maintains the total number of particles constant. However, the third step of the scheme introduced in the framework of a macroscopic description cannot be straightforwardly used in particle dynamics simulations in which the solvent would play the role of the reservoir $R_{2}$. Indeed, considering the step $B+S \rightleftharpoons 2 S$ would lead to the correct term proportional to $-B S$ but to an erroneous term proportional to $S^{2}$ instead of a constant in the rate equation of species B. Eventually, the chemical scheme is modified as follows [6]

$$
\begin{array}{lll}
\mathrm{A}+\mathrm{S} & \stackrel{k_{1}^{S}}{\longrightarrow} & 2 \mathrm{~S} \\
2 \mathrm{~A}+\mathrm{B} & \stackrel{k_{2}}{\longrightarrow} & 3 \mathrm{~A}
\end{array}
$$




$$
\begin{gathered}
\mathrm{B}+\mathrm{S} \\
\left\{\begin{array}{l}
\stackrel{k_{3}^{S}}{\longrightarrow} \\
\mathrm{R} \stackrel{k_{-3}}{\longrightarrow} \mathrm{B} \\
\mathrm{S} \longrightarrow \mathrm{R}
\end{array}\right.
\end{gathered}
$$

where the two last steps mean that $\mathrm{B}$ concentration is increased at the constant rate $k_{-3}=k_{-3}^{\prime} R$ while $\mathrm{S}$ concentration is decreased by the same amount to keep the total concentration $C$ constant. It is worth noting that the total concentration $C=A+B+S$ is conserved. The concentration $A$ and $B$ obey the partial differential equations:

$$
\begin{aligned}
& \frac{\partial A}{\partial t}=-k_{1} A\left[1-\frac{A+B}{C}\right]+k_{2} A^{2} B+D_{A} \frac{\partial^{2} A}{\partial x^{2}} \\
& \frac{\partial B}{\partial t}=k_{-3}-k_{3} B\left[1-\frac{A+B}{C}\right]-k_{2} A^{2} B+D_{B} \frac{\partial^{2} B}{\partial x^{2}}
\end{aligned}
$$

where the relation of conservation has been used to eliminate $S$ and the relations $k_{1}=k_{1}^{S} C$ and $k_{3}=k_{3}^{S} C$ between the rate constants introduced in Eqs. (1-3) and Eqs. (7-10) have been introduced. We straightforwardly verify that Eqs. $(11,12)$ converge towards the equations associated with the system not involving the solvent given in Eqs. (1-3) in the high dilution limit $\frac{A+B}{C} \longrightarrow 0$.

In a range of rate constants, the system given in Eqs. $(11,12)$ has three stationary states, two of which denoted by $\left(A^{T}, B^{T}\right)$ and $\left(A^{0}=0, B^{0}\right)$ are stable in the homogeneous case. When the ratio of the diffusion coefficients, $d=D_{B} / D_{A}$, is sufficiently large and for appropriate rate constant values, the steady state $\left(A^{T}, B^{T}\right)$ is destabilized by inhomogeneous perturbations and replaced by a periodic spatial structure of Turing type. The steady state $\left(A^{0}=0, B^{0}\right)$ remains stable and can be used to define a criterion evaluating the deviation from the dilution limit:

$$
\delta=\frac{A^{0}+B^{0}}{C}
$$

which remains unchanged for given parameter values, whereas $A+B$ may vary in space and time.

\section{ADAPTATION OF DSMC ALGORITHM}

The standard Direct Simulation Monte Carlo (DSMC) method [11, 12] has to be adapted to the simulation of a concentrated solution including two species A and B with sufficiently different diffusion coefficients, $D_{A}$ and $D_{B}$, and a solvent $\mathrm{S}$. The three kinds of particles $P=A, B, S$ are hard spheres and we use their radius $r_{P}$ as discriminating parameter. Collisions between three species are necessary to obtain different values of $D_{A}$ and $D_{B}$. Even in the most concentrated case considered, $\mathrm{S}$ is in excess with respect to $\mathrm{A}$ and $\mathrm{B}$ and the collisions between $\mathrm{A}$ and $\mathrm{B}$ can be neglected with respect to the collisions between $\mathrm{A}$ and $\mathrm{S}$ and the collisions between $\mathrm{B}$ and $\mathrm{S}$ as regards diffusion. Qualitatively, we impose $r_{B} \ll r_{S} \ll r_{A}$ and $S \gg A+B$ to obtain $D_{B}>D_{A}$, as required to stabilize the Turing structure. The precise conditions are given in references [2,6]. The diffusion coefficient $D_{P}$ of species $P=A, B$ obeys:

$$
D_{P}=\frac{3}{8(P+S)\left(r_{P}+r_{S}\right)^{2}} \sqrt{\frac{k_{B} T}{\pi m}}
$$

where $k_{B}$ is Boltzmann constant, $T$ is temperature, and $m$ is the mass of the hard spheres. It is worth noting that $D_{P}$ depends on $S$, i.e. on the dilution of the system. Roughly speaking, one has $D_{P} \propto \delta$, where $\delta$ is the deviation from the dilution limit defined in Eq. (13).

Another specificity of the system is the ternary reactive step given in Eq. (8) and associated with the autocatalytic formation of the activator A. We already address this issue in DSMC simulations of Schlögl model [5] and solved the problem as follows. A binary collision between A and B is performed with the usual condition on the relative velocity of the colliding pair leading to the formation of the short-lived complex AB. Then, the reaction between the complex 
$\mathrm{AB}$ and the nearest molecule $\mathrm{A}$ is accepted with a probability $A / C$, leading to the release of three $\mathrm{A}$ as expected. Hence, the rate constant $k_{2}$ obeys:

$$
k_{2}=\frac{4\left(r_{A}+r_{B}\right)^{2}}{C} \sqrt{\pi k_{B} T} m
$$

The specific expression of $k_{2}$ induces a dependence on $C$, i.e. on the deviation $\delta$ from the dilution limit. As for the diffusion coefficient $D_{P}$ with $P=A, B$, we have $k_{2} \propto \delta$.

The ternary step leads to a cubic term in the reaction-diffusion equations given in Eqs. $(11,12)$ which is necessary for the system to have three steady states. This last condition is required to observe trigger waves propagating between two homogeneous stable steady states, one of which may be destabilized by inhomogeneous perturbations leading to a Turing pattern. We exploit this property in the choice of original boundary conditions inspired by the formation of the spine in vertebrate embryos and introduced here by biomimicry $[9,13,14,3]$. The great advantage of considering a propagating front in a growing system is to avoid fixed boundary conditions, known to perturb the wavelength of the Turing pattern. Indeed, using DSMC for zero-flux boundary conditions in a small system with a length slightly smaller than two wavelengths, transitions between Turing patterns developing over one and a half wavelength or two wavelengths can be observed [2]. This phenomenon could affect the estimation of the wavelength of the structure, whereas considering a Turing pattern developing between zero-flux boundary conditions at one end and a propagating wave front ensures the reproducibility of wavelength determination.

\section{TURING PATTERNS WITH TUNABLE WAVELENGTH}

We choose a step function as initial condition with the first 50 cells on the left prepared in the steady state $\left(A^{T}, B^{T}\right)$ and the remaining cells prepared in the steady state $\left(A^{0}, B^{0}\right)$. Typical profiles for A and B species deduced from the numerical integration of Eqs. $(11,12)$ and DSMC simulations are given in Fig. 1. A Turing structure with a well-
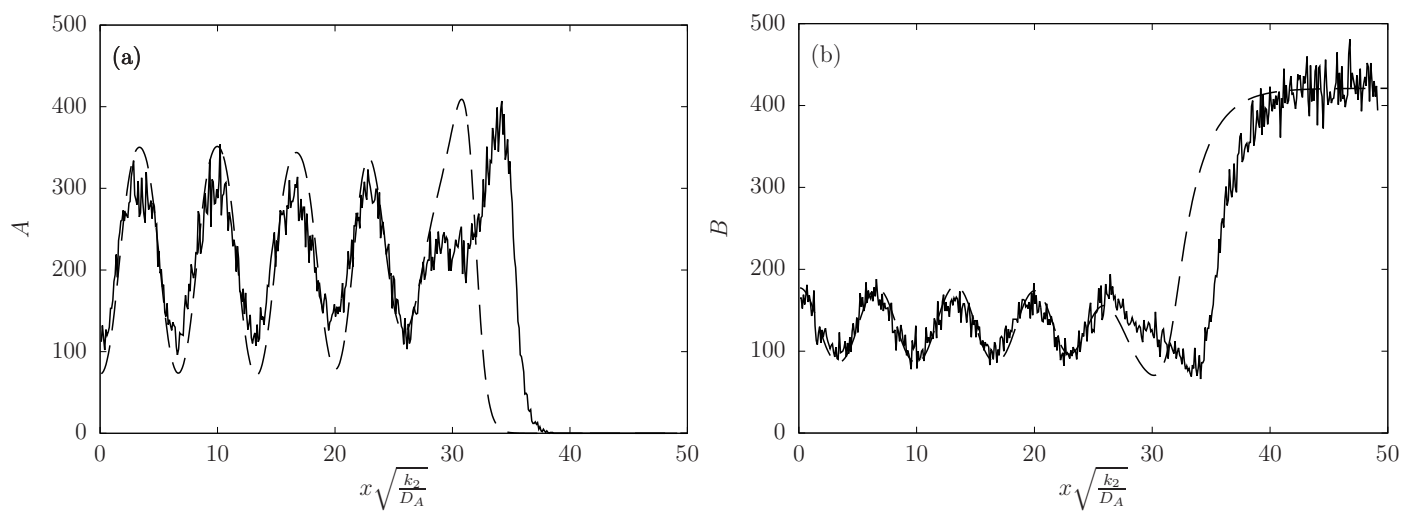

FIGURE 1. Concentration profiles deduced from the numerical solution of Eqs. $(11,12)$ (dashed line) and number of particles per cell in simulations (solid line) versus scaled spatial coordinate $x \sqrt{\frac{k_{2}}{D_{A}}}$ for Eqs. (7-10), for a given deviation $\delta=0.05$ from the dilution limit, at scaled time $k_{2} t=6986$. Snapshot of the profiles for species A (a) and B (b). The parameters take the following values: $\frac{k_{1}}{k_{2}}=2.9 \times 10^{4}, \frac{k_{3}}{k_{2}}=2.2 \times 10^{4}, \frac{k_{-3}}{k_{2}}=8.8 \times 10^{6}, d=10$.

defined wavelength $\lambda$ develops behind a wave front propagating to the right for both approaches. The main question is: Does $\lambda$ vary as the deviation $\delta$ from the dilution limit or equivalently the total concentration $C$ varies ?

Before answering the question, we indirectly check the ability of DSMC simulations to properly reproduce the rate constant values for different deviations from the dilution limit. Figure 2 gives the variation of the steady state $\left(A^{T}, B^{T}\right)$ versus the deviation $\delta$ from the dilution limit. The prediction of the macroscopic approach based on Eqs. $(11,12)$ compares well with the spatially-averaged numbers $N_{A^{T}}$ and $N_{B^{T}}$ of particles A and B deduced from DSMC. The average is performed over four wavelengths. The simulation results are not displayed for $\delta=0.01$ due to the computation cost: In this case, the total number of particles per cell would typically reach 40400 for the chosen parameter values. 


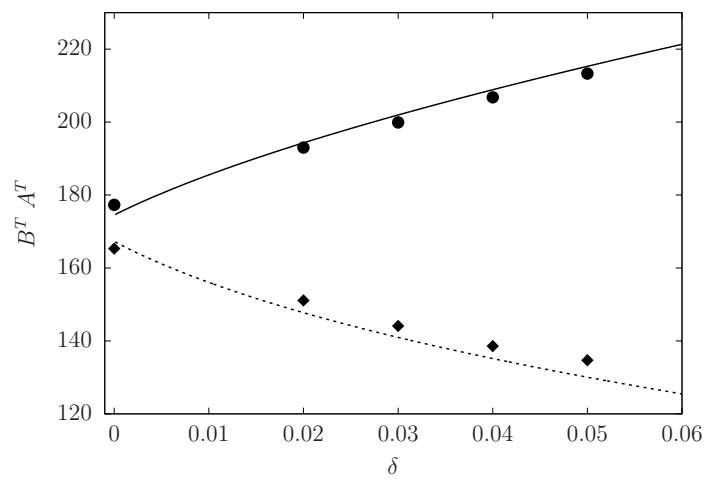

FIGURE 2. Macroscopic prediction of the steady state concentrations $A^{T}$ (solid line) and $B^{T}$ (dotted line) versus the deviation $\delta$ from the dilution limit. Circles (diamonds, resp.) give the mean number of A (B, resp.) particles per cell in the region of the simulated Turing pattern. For $\delta \neq 0$, the mechanism given in Eqs. (7-10) is used whereas the mechanism given in Eqs. (1-3) is used for $\delta=0$. The value of the other parameters is given in caption of Fig. 1 .

We compare the variations of the scaled wavelength $\lambda \sqrt{\frac{k_{2}}{D_{A}}}$ of Turing pattern versus $\delta$ deduced from Eqs. $(11,12)$ and DSMC results in Fig. 3.

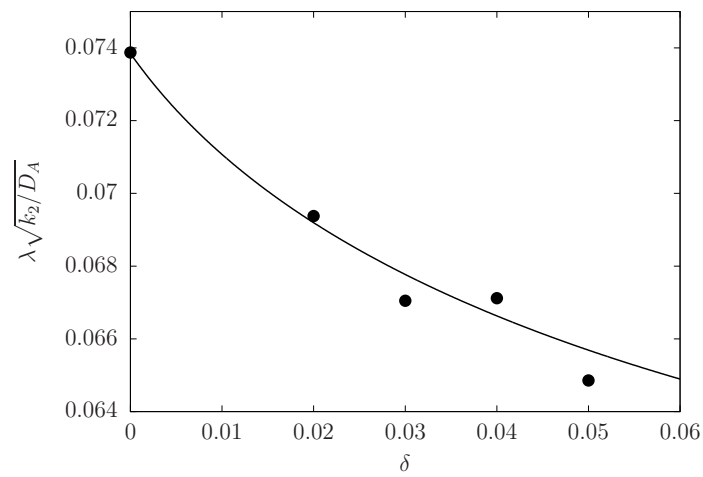

FIGURE 3. Macroscopic prediction of the scaled wavelength of the Turing pattern (solid line) and scaled wavelength deduced from the simulations (circles) versus deviation $\delta$ from the dilution limit. For $\delta \neq 0$, the mechanism given in Eqs. (7-10) is used whereas the mechanism given in Eqs. (1-3) is used for $\delta=0$. The value of the other parameters is given in caption of Fig. 1.

Indeed, the scaled wavelength depends on ratios of rate constants and diffusion coefficients. These ratios are set in all the simulations to the same values given in the caption of Fig. 1 and do not vary with the deviation $\delta$ from the dilution limit. Knowing that $D_{A}$ and $k_{2}$ are constant in the macroscopic approach, we introduce the scaled wavelength to isolate the intrinsic dependence on $\delta$ introduced by the role of the solvent in the chemical mechanism, regardless of the specific expressions of the parameters $D_{A}$ (Eq. (14)) and $k_{2}$ (Eq. (15)) in the microscopic approach. As shown in Fig. 3, the agreement between the microscopic and the macroscopic descriptions is very good, which validates that DSMC correctly reproduce the ratio of diffusion coefficients. Simulation results become more noisy as $\delta$ increases, in agreement with the loss of stability of Turing structure predicted by Eqs. $(11,12)$ [6]. DSMC results are obtained for $\delta=0$ by considering the chemical mechanism without explicit role of the solvent given in Eqs. (1-3). We have checked that, for $\delta=0$, the same result was obtained for all the considered values of $S$.

Figure 4 gives the answer to the main question raised in this work. The wavelength of a Turing pattern may be tuned in particle dynamics simulations by varying the total concentration of solvent, i.e. the deviation $\delta$ from the dilution limit. Typically, for the chosen parameter values, the wavelength goes from 26 to 17 when $\delta$ goes from 0.02 to 0.04 . Hence, increasing the deviation from the dilution limit by a factor 2 leads to a decrease of the wavelength by a factor greater than 1.5. The simulation results are given in arbitrary units. The conversion of the wavelength in nanometers is easy. For example, for $\delta=0.05$ and small molecules A and B in water S, we find a wavelength of the order of $200 \mathrm{~nm}$ 


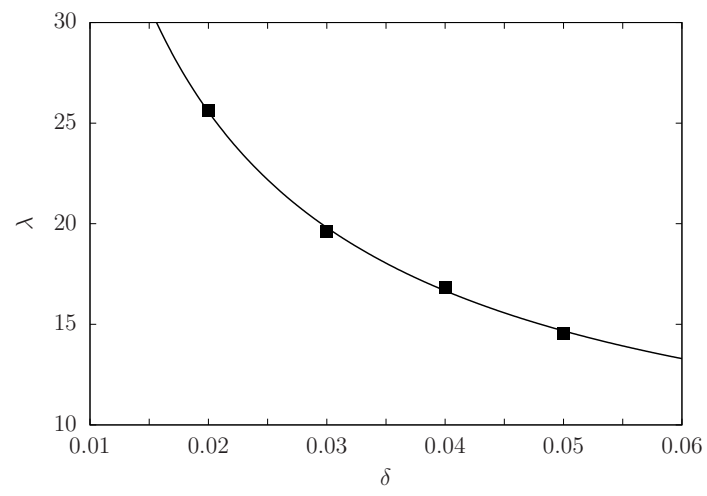

FIGURE 4. Same caption as in Fig. 3 without scaling of the wavelength.

[6]. Hence, DSMC reveals the possibility to tune the wavelength of Turing pattern on a submicrometric scale, simply by playing on the dilution of the system. The application to chemical engineering nevertheless requires the control of a chemical system whose kinetics explicitly involves the solvent, for example as made precise in the mechanism given in Eqs. (4-6).

\section{CONCLUSION}

We have reported on a reaction-diffusion model involving the reactive role of the solvent and proved that dilution could be used to control the wavelength of a Turing pattern. The direct simulation Monte Carlo (DSMC) method has been successfully extended to concentrated solutions. The simulation results show that it is possible to monitor Turing patterns at a submicrometric scale, simply by changing the total concentration: It is sufficient to increase concentration by a factor 2 to obtain a wavelength reduction by the same factor. The results give some indications to design a chemical scheme allowing experimental validation in the framework of chemical engineering.

\section{ACKNOWLEDGMENTS}

This publication is part of a project that has received funding from the European Union's Horizon 2020 (H2020EU.1.3.4.) research and innovation program under the Marie Skłodowska-Curie Actions (MSCA-COFUND ID 711859) and from the Polish Ministry of Science and Higher Education for the implementation of an international cofinanced project.

\section{REFERENCES}

[1] A. M. Turing, Philos. Trans. R. Soc. London, Ser. B 237, 37 (1952).

[2] P. Dziekan, A. Lemarchand, and B. Nowakowski, J. Chem. Phys. 137, 074107 (2012).

[3] L. Signon, B. Nowakowski, and A. Lemarchand, Phys. Rev. E 93, 042402 (2016).

[4] M. Mareschal and A. De Wit, J. Chem. Phys. 96, 2000 (1992).

[5] A. Lemarchand and B. Nowakowski, Physica A 271, 87 (1999).

[6] G. Morgado, B. Nowakowski, and A. Lemarchand, Phys. Rev. E 98, 032213 (2018).

[7] J. Schnakenberg, J. Theor. Biol. 81, 389 (1979).

[8] P. Gray and S. K. Scott, Chem. Eng. Sci. 39, 1087 (1984).

[9] A. Lemarchand and B. Nowakowski, EPL 94, 48004 (2011).

[10] A. Gierer and H. Meinhardt, Kybernetik 12, 30 (1972).

[11] G. A. Bird, Molecular Gas Dynamics and the Direct Simulation of Gas Flows (Clarendon, Oxford, 1994).

[12] F. J. Alexander and A. L. Garcia, Comput. Phys. 11, 588 (1997).

[13] P. Dziekan, L. Signon, B. Nowakowski, and A. Lemarchand, J Chem. Phys. 139, 114107 (2013).

[14] P. Dziekan, L. Signon, B. Nowakowski, and A. Lemarchand, Commun. Theor. Phys. 62, 622 (2014). 